\title{
Topological twisted sigma model with H-flux revisited
}

\author{
Wu-yen Chuang \\ ITP, Stanford University, Stanford, CA 94305, USA \\ SLAC, Stanford University, Menlo Park, CA 94025, USA \\ wychuang@stanford.edu
}

\begin{abstract}
In this paper we revisit the topological twisted sigma model with H-flux. We explicitly expand and then twist the worldsheet Lagrangian for bi-Hermitian geometry. we show that the resulting action consists of a BRST exact term and pullback terms, which only depend on one of the two generalized complex structures and the B-field. We then discuss the topological feature of the model.
\end{abstract}

\section{Introduction}

It is a very convenient and powerful approach to obtain topological field theories by twisting supersymmetric field theory [1]. It was furthur shown that the $N=(2,2)$ worldsheet sigma model with the Kähler target space admits A and B types of twisting [2]. However the Kähler condition is not crucial to perform the A and B twists. What is really needed is to have $N=(2,2)$ worldsheet supersymmetry so that $U(1)_{V}$ and $U(1)_{A}$ exist.

From the viewpoint of the $N=(2,2)$ worldsheet supersymmetry algebra the twists are achieved by replacing the $2 \mathrm{~d}$ Euclideanized spacetime rotation group $U(1)_{E}$ with the diagonal subgroup of $U(1)_{E} \times U(1)_{R}$, where $U(1)_{R}$ is either $U(1)_{V}$ or $U(1)_{A}$ R-symmetry in the $N=(2,2)$ supersymmetry group.

In 1984 the most general geometric backgrounds for $N=(2,2)$ supersymmetric sigma models was proposed by Gates, Hull, and Roček [4]. The geometric backgrounds (a.k.a. bi-Hermitian geometry) consists of a set of data $\left(J_{+}, J_{-}, g, H\right) . \quad J_{ \pm}$are two different integrable complex structures and the metric $g$ is Hermitian with respect to either one 
of $J_{ \pm}$. Moreover $J_{ \pm}$are convariantly constant with respect to the torsional connections $\Gamma \pm g^{-1} H$, where $H$ is a closed 3 -form on the manifold. The manifold is apparently non-Kähler due to the presence of the torsions.

Bi-Hermitian geometry started to re-receive new attention after Hitchin introduced the notion of generalized geometry [6] and Gualtieri furthur showed that the geometry is equivalent to a pair of commuting (twisted) generalized complex ((T)GC for short) structures on the manifold $M$, namely, the twisted generalized Kähler structure [7].

Since the worldsheet theory with bi-Hermitian target has $N=(2,2)$ supersymmetry, we definitely can consider its topological twisted models. In [5] Kapustin and Li considered such a topological model and showed that on the classical level the topological observables in a given twisted model correspond to the Lie algebroid cohomologies associated with one of the two twisted generalized complex structures. The same problem was also considered by many other authors from Hamiltonian approach or using Batalin-Vilkovisky quantization [8] [9] [10].

Although it is definitely true that the twisted models for bi-Hermitian geometries are topological, the explicit construction of the twisted Lagrangian is lacking. The difficulties of such a calculation lie in that people are so accustomed to using complex geometry that they feel relunctant to perform a calculation which needs to be done in the real coordinate basis with projectors. A priori, we should be able to express the twisted Lagrangian for the generalized geometry as some BRST exact piece plus certain pullback terms which only depend on one of the twisted generalized complex structures.

By the end of the paper we will see that this is indeed true. However since the pullback object is not closed it is not clear that the action is topological. This issue is made clear in [15]. The paper is organized as follows. In Section 2 we first review the sigma models with Riemannian and Kähler targets and discuss the properties of the twisted Lagrangian. In Section 3 we present the computation of the twisted topological models for bi-Hermitian geometries and express the twisted Lagrangian in the aforementioned way. In section 4 we conclude, discuss the limitation of the twisted models, and mention some open questions. Some basics and definitions of the generalized geometry will be presented in the appendix. 


\section{Topological sigma model with Kähler targets}

We first recall some basic facts about the worldsheet sigma models with Riemannian or Kähler manifolds as targets. Throughout the whole paper lowercase English letters $a, b, c, \ldots$ are indices for the real coordinates on the targets, while Greek letter $\mu, \nu, \sigma, \ldots$ are those for holomorphic coordinates. (And of course $\bar{\mu}, \bar{\nu}, \bar{\sigma}, \ldots$ for antiholomorphic coordiantes.) Although it has been shown that the off-shell formalism exists even for the bi-Hermitian geometry [11], we will only work in the on-shell supersymmetry formalism to simplify the calculation.

The nonlinear sigma model with a Riemannian manifold $M$ has natural $(1,1)$ worldsheet supersymmetric formalism. The model is governed by an embedding map $\Phi: \Sigma \rightarrow$ $M$ where $\Sigma$ is a Riemann surface. The Lagrangian is

$$
L=2 t \int d^{2} z d^{2} \theta g_{a b}(\Phi) D_{+} \Phi^{a} D_{-} \Phi^{b}
$$

where

$$
\begin{gathered}
D_{ \pm}=\frac{\partial}{\partial \theta^{ \pm}}+i \theta^{ \pm}\left(\frac{\partial}{\partial x^{0}} \pm \frac{\partial}{\partial x^{1}}\right) \\
\Phi^{a}=\phi^{a}+\theta^{+} \psi_{+}^{a}+\theta^{-} \psi_{-}^{a}+\theta^{-} \theta^{+} F^{a} \\
d^{2} z=\frac{i}{2} d z \wedge d \bar{z}
\end{gathered}
$$

Exapnding out (2.1) and then setting $F^{a}=\Gamma_{b c}^{a} \psi_{+}^{b} \psi_{-}^{c}$ (the on-shell value of $F^{a}$ ) we have

$$
\begin{aligned}
L=2 t \int d^{2} z \quad(\quad & \frac{1}{2} g_{a b} \partial_{z} \phi^{a} \partial_{\bar{z}} \phi^{b}+\frac{i}{2} g_{a b} \psi_{-}^{a} D_{z} \psi_{-}^{b} \\
& \left.+\frac{i}{2} g_{a b} \psi_{+}^{a} D_{\bar{z}} \psi_{+}^{b}+\frac{1}{4} R_{a b c d} \psi_{+}^{a} \psi_{+}^{b} \psi_{-}^{c} \psi_{-}^{d}\right)
\end{aligned}
$$

where $D_{\bar{z}} \psi_{+}^{a}=\partial_{\bar{z}} \psi_{+}^{a}+\Gamma_{b c}^{a} \partial_{\bar{z}} \phi^{b} \psi_{+}^{c}$ and $D_{z} \psi_{-}^{a}=\partial_{z} \psi_{-}^{a}+\Gamma_{b c}^{a} \partial_{z} \phi^{b} \psi_{-}^{c}$.

If the target space is Kähler the nonlinear sigma model will have an additional $(1,1)$ supersymmetry, turning the theory into $N=(2,2)$ sigma model [3]. The Lagrangian of such a sigma model is written as

$$
\begin{aligned}
L=2 t \int d^{2} z \quad(\quad & \frac{1}{2} g_{a b} \partial_{z} \phi^{a} \partial_{\bar{z}} \phi^{b}+i g_{\bar{\mu} \mu} \psi_{-}^{\bar{\mu}} D_{z} \psi_{-}^{\mu} \\
& \left.+i g_{\bar{\mu} \mu} \psi_{+}^{\bar{\mu}} D_{\bar{z}} \psi_{+}^{\mu}+R_{\mu \bar{\mu} \nu \bar{\nu}} \psi_{+}^{\mu} \psi_{+}^{\bar{\mu}} \psi_{-}^{\nu} \psi_{-}^{\bar{\nu}}\right)
\end{aligned}
$$


The detailed supersymmetry transformations are listed as follows [2].

$$
\begin{aligned}
& \delta \phi^{\mu}=i \epsilon_{-} \psi_{+}^{\mu}+i \epsilon_{+} \psi_{-}^{\mu} \\
& \delta \phi^{\bar{\mu}}=i \bar{\epsilon}_{-} \psi_{+}^{\bar{\mu}}+i \bar{\epsilon}_{+} \psi_{-}^{\bar{\mu}} \\
& \delta \psi_{+}^{\mu}=-\bar{\epsilon}_{-} \partial_{z} \phi^{\mu}-i \epsilon_{+} \psi_{-}^{\nu} \Gamma_{\nu \sigma}^{\mu} \psi_{+}^{\sigma} \\
& \delta \psi_{+}^{\bar{\mu}}=-\epsilon_{-} \partial_{z} \phi^{\bar{\mu}}-i \bar{\epsilon}_{+} \psi_{-}^{\bar{\nu}} \Gamma_{\bar{\nu} \bar{\sigma}}^{\bar{\mu}} \psi_{+}^{\bar{\sigma}} \\
& \delta \psi_{-}^{\mu}=-\bar{\epsilon}_{+} \partial_{\bar{z}} \phi^{\mu}-i \epsilon_{-} \psi_{+}^{\nu} \Gamma_{\nu \sigma}^{\mu} \psi_{-}^{\sigma} \\
& \delta \psi_{-}^{\bar{\mu}}=-\epsilon_{+} \partial_{\bar{z}} \phi^{\bar{\mu}}-i \bar{\epsilon}_{-} \psi_{+}^{\bar{\nu}} \Gamma_{\bar{\nu} \bar{\sigma}}^{\bar{\mu}} \psi_{-}^{\bar{\sigma}}
\end{aligned}
$$

\section{$2.1 \quad$ Kähler A model}

An A-twist will turn $\psi_{+}^{\mu}$ and $\psi_{-}^{\bar{\mu}}$ into sections of $\Phi^{*}\left(T^{1,0} X\right)$ and $\Phi^{*}\left(T^{0,1} X\right)$, denoted as $\chi^{\mu}$ and $\chi^{\bar{\mu}}$. And $\psi_{+}^{\bar{\mu}}$ and $\psi_{-}^{\mu}$ become sections of $\Omega_{\Sigma}^{1,0} \otimes \Phi^{*}\left(T^{0,1} X\right)$ and $\Omega_{\Sigma}^{0,1} \otimes \Phi^{*}\left(T^{1,0} X\right)$, denoted as $\psi_{z}^{\bar{\mu}}$ and $\psi_{\bar{z}}^{\mu}$. In order to get the transformation laws we simply set $\epsilon_{+}=\bar{\epsilon}_{-}=0$ in (2.7). After A-twist the Lagrangian becomes

$$
\begin{aligned}
L=2 t \int d^{2} z \quad( & \frac{1}{2} g_{a b} \partial_{z} \phi^{a} \partial_{\bar{z}} \phi^{b}+i g_{\bar{\mu} \mu} \psi_{z}^{\bar{\mu}} D_{\bar{z}} \chi^{\mu} \\
& \left.+i g_{\bar{\mu} \mu} \psi_{\bar{z}}^{\mu} D_{z} \chi^{\bar{\mu}}-R_{\mu \bar{\mu} \nu \bar{\nu}} \psi_{\bar{z}}^{\mu} \psi_{z}^{\bar{\mu}} \chi^{\nu} \chi^{\bar{\nu}}\right)
\end{aligned}
$$

The key fact as stated in [2] is that the Lagrangian can be recast into a very suggestive form, which is a BRST exact term plus a pullback term depdending only on the Kähler structure of the target space. Upon deriving this the equatoins of motion of $\psi$ are needed.

$$
L=i t \int d^{2} z\left\{Q, V_{A}\right\}+t \int \Phi^{*}(K)
$$

with $V_{A}=g_{\mu \bar{\nu}}\left(\psi_{z}^{\bar{\nu}} \partial_{\bar{z}} \phi^{\mu}+\partial_{z} \phi^{\bar{\nu}} \psi_{\bar{z}}^{\mu}\right)$ and $K=-i g_{\mu \bar{\nu}} d z^{\mu} d z^{\bar{\nu}}$. From this expression we realize

that the Kähler A model depends only on the cohomology class of $K$. $\int \Phi^{*}(K)$ also depends on the homotopy class of the mapping $\Phi$, but in the path integral all the homotopy classes will be summed over.

\subsection{Kähler B model}

We also recall some basics about the Kähler B model which will be useful later. The B twist will turn $\psi_{ \pm}^{\bar{\mu}}$ into sections of $\Phi^{*}\left(T^{0,1} X\right)$, and $\psi_{+}^{\mu}$ and $\psi_{-}^{\mu}$ into sections of $\Omega_{\Sigma}^{1,0} \otimes$ 
$\Phi^{*}\left(T^{0,1} X\right)$ and $\Omega_{\Sigma}^{0,1} \otimes \Phi^{*}\left(T^{0,1} X\right)$ respectively. The transformation can be written as

$$
\begin{aligned}
& \delta \phi^{\mu}=0 \\
& \delta \phi^{\bar{\mu}}=i \epsilon \eta^{\bar{\mu}} \\
& \delta \eta^{\bar{\mu}}=\delta \theta_{\mu}=0 \\
& \delta \rho^{\mu}=-\epsilon d \phi^{\mu}
\end{aligned}
$$

where

$$
\begin{aligned}
\eta^{\bar{\mu}} & =\psi_{+}^{\bar{\mu}}+\psi_{-}^{\bar{\mu}} \\
\theta_{\mu} & =g_{\mu \bar{\mu}}\left(\psi_{+}^{\bar{\mu}}-\psi_{-}^{\bar{\mu}}\right) \\
\rho^{\mu} & =\psi_{+}^{\mu}+\psi_{-}^{\mu}
\end{aligned}
$$

After the B twisting the Lagrangian explicitly becomes

$$
\begin{aligned}
L=t \int d^{2} z \quad(\quad & g_{a b} \partial_{z} \phi^{a} \partial_{\bar{z}} \phi^{b}+i g_{\bar{\mu} \mu} \eta^{\bar{\mu}}\left(D_{z} \rho_{\bar{z}}^{\mu}+D_{\bar{z}} \rho_{z}^{\mu}\right) \\
& \left.+i \theta_{\mu}\left(D_{\bar{z}} \rho_{z}^{\mu}-D_{z} \rho_{\bar{z}}^{\mu}\right)+R_{\mu \bar{\mu} \nu \bar{\nu}} \rho_{z}^{\mu} \rho_{\bar{z}}^{\nu} \eta^{\bar{\mu}} \theta_{\sigma} g^{\sigma \bar{\nu}}\right)
\end{aligned}
$$

which can be reexpressed as follows.

$$
L=i t \int\left\{Q, V_{B}\right\}+t W
$$

where

$$
W=\int_{\Sigma}\left(-\theta_{\mu} D \rho^{\mu}-\frac{i}{2} R_{\mu \bar{\mu} \nu \bar{\nu}} \rho^{\mu} \wedge \rho^{\nu} \eta^{\bar{\mu}} \theta_{\sigma} g^{\sigma \bar{\nu}}\right)
$$

and the $D$ operator is the exterior derivative on the worldsheet $\Sigma$ by using the pullback of the Levi-Civita connection on $M$. The model is topological because it is independent of the complex structure of the worldsheet and the Kähler structure of the target space. However the model do depend on the complex structure, which can be seen from the BRST variations of the fields.

\section{Bi-Hermitian geometry and its topological twisted models}

As stated in the introduction the most general $(2,2)$ nonlinear sigma model with $H$ is described in [4], which is also known as "bi-Hermitian geometry." We will simply quote 
the properties of the geometry, without any derivations of the requirements. With the non-trivial B-field turned on, the worldsheet action is given by

$$
L=2 t \int d^{2} z d^{2} \theta\left(g_{a b}(\Phi)+b_{a b}(\Phi)\right) D_{+} \Phi^{a} D_{-} \Phi^{b}
$$

The first set of $(1,1)$ supersymmetry is as usual while the additional $(1,1)$ supersymmetry transformations are given by two different complex structures

$$
\begin{array}{r}
\delta^{1} \Phi^{a}=i \epsilon_{+}^{1} D_{+} \Phi^{a}+i \epsilon_{-}^{1} D_{-} \Phi^{a} \\
\delta^{2} \Phi^{a}=i \epsilon_{+}^{2} D_{+} \Phi^{b} J_{+b}^{a}+i \epsilon_{-}^{2} D_{-} \Phi^{b} J_{-b}^{a}
\end{array}
$$

where $J_{+}$and $J_{-}$are the complex structures seen by the left and right movers respectively. Requiring (3.1) to be invariant under the transformations leads us to the conditions:

$$
J_{ \pm}^{t} g J_{ \pm}=g \quad \nabla^{ \pm} J_{ \pm}=0
$$

where $\nabla^{ \pm}$are the covariant derivatives with torsional connections $\Gamma_{ \pm}=\Gamma \pm g^{-1} H$. The first condition implies that the metric is Hermitian with respect to the either one of the complex structures $J_{ \pm}$. And the second condition in (3.3) explicitly becomes

$$
J_{ \pm b, c}^{a}=\Gamma_{ \pm c b}^{d} J_{ \pm d}^{a}-\Gamma_{ \pm c d}^{a} J_{ \pm b}^{d}
$$

Equation (3.4) will be used when we try to contruct the generalized A/B models in real coordinate basis. Moreover the $H$ field is of type $(2,1)+(1,2)$ with respect to both complex structures $J_{ \pm}$. Expanding (3.1) out and then setting $F^{a}$ to its on-shell value we have the following worldsheet action in component fields

$$
\begin{aligned}
F^{a} & =\Gamma_{+b c}^{a} \psi_{+}^{b} \psi_{-}^{c}=-\Gamma_{-b c}^{a} \psi_{-}^{b} \psi_{+}^{c} \\
L & =2 t \int d^{2} z\left(\frac{1}{2}\left(g_{a b}+b_{a b}\right) \partial_{z} \phi^{a} \partial_{\bar{z}} \phi^{b}+\frac{i}{2} g_{a b}\left(\psi_{-}^{a} \partial_{z} \psi_{-}^{b}+\psi_{+}^{a} \partial_{\bar{z}} \psi_{+}^{b}\right)\right. \\
& \left.+\frac{i}{2} \psi_{-}^{a} \partial_{z} \phi^{b} \psi_{-}^{c}\left(\Gamma_{a b c}-H_{a b c}\right)+\frac{i}{2} \psi_{+}^{a} \partial_{\bar{z}} \phi^{b} \psi_{+}^{c}\left(\Gamma_{a b c}+H_{a b c}\right)+\frac{1}{4} R_{+a b c d} \psi_{+}^{a} \psi_{+}^{b} \psi_{-}^{c} \psi_{-}^{d}\right)
\end{aligned}
$$

where $R_{+a b c d}$ is the curvature of the torsional connection $\Gamma_{+b c}^{a}$. 


$$
R_{ \pm a b c d}=R_{a b c d} \pm \frac{1}{2}\left(\nabla_{d} H_{a b c}-\nabla_{c} H_{a b d}\right)+\frac{1}{4}\left(H_{a d}^{e} H_{e b c}-H_{a c}^{e} H_{e b d}\right)
$$

Since the theory is of $(2,2)$ type there exist two $U(1)$ R-symmetries for the worldsheet fermions, $U(1)_{V}$ and $U(1)_{A}[5]$. The topological A and B twists will shift the spins of the fermions by the charges of $U(1)_{V}$ and $U(1)_{A}$ respectively. The charge assignments are worked out in [5] and [13].

$$
\begin{array}{lll}
U(1)_{V}: & q_{V}\left(\bar{P}_{+} \psi_{+}\right)=-1 & q_{V}\left(\bar{P}_{-} \psi_{-}\right)=-1 \\
U(1)_{A}: & q_{A}\left(\bar{P}_{+} \psi_{+}\right)=-1 & q_{V}\left(\bar{P}_{-} \psi_{-}\right)=+1
\end{array}
$$

with the following projectors defined for conveniences.

$$
P_{ \pm}=\frac{1}{2}\left(1+i J_{ \pm}\right), \quad \bar{P}_{ \pm}=\frac{1}{2}\left(1-i J_{ \pm}\right)
$$

Moreover the $U(1)$ R-symmetry used in the topological twist needs to be non-anomalous. The anomalies are computed by Atiyah-Singer index theorem and the conditions are

$$
\begin{array}{ll}
U(1)_{V}: & c_{1}\left(T_{-}^{1,0}\right)-c_{1}\left(T_{+}^{1,0}\right)=0 \\
U(1)_{A}: & c_{1}\left(T_{-}^{1,0}\right)+c_{1}\left(T_{+}^{1,0}\right)=0
\end{array}
$$

Using the language of generalized complex geometry we have two commuting twisted generalized complex structures $\left(\mathcal{J}_{1}, \mathcal{J}_{2}\right)$. $\mathcal{J}_{1}$ and $\mathcal{J}_{2}$ are endomorphisms on $T M \oplus T^{*} M$, which square to -1 . Let $E_{1}$ and $E_{2}$ be the $i$-eigenbundles of $\mathcal{J}_{1}$ and $\mathcal{J}_{2}$. The conditions can be repackaged into

$$
\begin{array}{ll}
U(1)_{V}: & c_{1}\left(E_{2}\right)=0 \\
U(1)_{A}: & c_{1}\left(E_{1}\right)=0
\end{array}
$$

The supersymmetry transformation laws can be derived from (3.2).

$$
\begin{aligned}
& \delta_{+}^{1} \phi=\psi_{+} \quad \delta_{-}^{1} \phi=\psi_{-} \quad \delta_{+}^{2} \phi=J_{+} \psi_{+} \quad \delta_{-}^{2} \phi=J_{-} \psi_{-} \\
& \delta_{+}^{1} \psi_{+}=-i \partial_{z} \phi \quad \delta_{-}^{1} \psi_{+}=F \quad \delta_{+}^{2} \psi_{+}=i J_{+} \partial_{z} \phi \quad \delta_{-}^{2} \psi_{+}=J_{-} F \\
& \delta_{+}^{1} \psi_{-}=-F \quad \delta_{-}^{1} \psi_{-}=-i \partial_{\bar{z}} \quad \delta_{+}^{2} \psi_{-}=-J_{+} F \quad \delta_{-}^{2} \psi_{-}=i J_{-} \partial_{\bar{z}} \phi
\end{aligned}
$$


We can then define the linear combinations of the supersymmetry generators.

$$
\begin{aligned}
Q_{+}=\frac{1}{2}\left(Q_{+}^{1}+i Q_{+}^{2}\right) & \bar{Q}_{+}=\frac{1}{2}\left(Q_{+}^{1}-i Q_{+}^{2}\right) \\
Q_{-}=\frac{1}{2}\left(Q_{-}^{1}+i Q_{-}^{2}\right) & \bar{Q}_{-}=\frac{1}{2}\left(Q_{-}^{1}-i Q_{-}^{2}\right)
\end{aligned}
$$

We then express the on-shell variation laws in the following forms

$$
\begin{aligned}
\delta \phi^{a} & =i\left(\epsilon_{+}\left(P_{+} \psi_{+}\right)^{a}+\bar{\epsilon}_{+}\left(\bar{P}_{+} \psi_{+}\right)^{a}\right)+i\left(\epsilon_{-}\left(P_{-} \psi_{-}\right)^{a}+\bar{\epsilon}_{-}\left(\bar{P}_{-} \psi_{-}\right)^{a}\right) \\
\delta \psi_{+} & =-\epsilon_{+}\left(\bar{P}_{+} \partial_{z} \phi\right)^{a}-\bar{\epsilon}_{+}\left(P_{+} \partial_{z} \phi\right)^{a}-\Gamma_{+b c}^{a} \delta \phi^{b} \psi_{+}^{c} \\
& +i H_{b c}^{a}\left(\epsilon_{+}\left(P_{+} \psi_{+}\right)^{b}+\bar{\epsilon}_{+}\left(\bar{P}_{+} \psi_{+}\right)^{b}\right) \psi_{+}^{c}-\frac{i}{2}\left(\epsilon_{+} P_{+d}^{a}+\bar{\epsilon}_{+} \bar{P}_{+d}^{a}\right) H_{b c}^{d} \psi_{+}^{b} \psi_{+}^{c} \\
\delta \psi_{-} & =-\epsilon_{-}\left(\bar{P}_{-} \partial_{z} \phi\right)^{a}-\bar{\epsilon}_{-}\left(P_{-} \partial_{z} \phi\right)^{a}-\Gamma_{-b c}^{a} \delta \phi^{b} \psi_{-}^{c} \\
& +i H_{b c}^{a}\left(\epsilon_{-}\left(P_{-} \psi_{-}\right)^{b}+\bar{\epsilon}_{-}\left(\bar{P}_{-} \psi_{-}\right)^{b}\right) \psi_{-}^{c}-\frac{i}{2}\left(\epsilon_{-} P_{-d}^{a}+\bar{\epsilon}_{-} \bar{P}_{-d}^{a}\right) H_{b c}^{d} \psi_{-}^{b} \psi_{-}^{c}
\end{aligned}
$$

where $\epsilon_{ \pm}$are the variation parameters of $Q_{ \pm}$.

The BRST operators for the generalized A and B models can be taken as:

$$
Q_{A}=Q_{+}+\bar{Q}_{-}, \quad Q_{B}=\bar{Q}_{+}+\bar{Q}_{-} .
$$

Before the topological twists we have the worldsheet fermions $P_{+} \psi_{+}, \bar{P}_{+} \psi_{+}, P_{-} \psi_{-}$, and $\bar{P}_{-} \psi_{-}$. These fermions are sections of certain bundles. For instance $\bar{P}_{+} \psi_{+}$is a section of $K^{1 / 2} \otimes \Phi^{*}\left(T_{+}^{0,1} X\right)$ where $K$ is the canonical line bundle of the worldsheet (the bundle of $(1,0)$ form.) and $T_{+}^{0,1}$ is the $(0,1)$ part of the tangent bundle with respect to $J_{+}$. After performing topological A-twist, the spins of the fermions will be changed as follows.

$$
\begin{aligned}
&\left(P_{+} \psi_{+}\right)^{a} \equiv \chi^{a} \in \Gamma\left(\Phi^{*}\left(T_{+}^{1,0} X\right)\right) \\
&\left(\bar{P}_{+} \psi_{+}\right)^{a} \equiv \chi_{z}^{a} \in \Gamma\left(\Omega_{\Sigma}^{(1,0)} \otimes \Phi^{*}\left(T_{+}^{0,1} X\right)\right) \\
&\left(P_{-} \psi_{-}\right)^{a} \equiv \lambda_{\bar{z}}^{a} \in \Gamma\left(\Omega_{\Sigma}^{(0,1)} \otimes \Phi^{*}\left(T_{-}^{1,0} X\right)\right) \\
&\left(\bar{P}_{-} \psi_{-}\right)^{a} \equiv \lambda^{a} \in \Gamma\left(\Phi^{*}\left(T_{-}^{0,1} X\right)\right)
\end{aligned}
$$

On the other hand the B-twist case can be obtained similarly. For completeness we list the sections in the generalized B-model with the BRST charge $Q_{B}=\bar{Q}_{+}+\bar{Q}_{-}$. 


$$
\begin{aligned}
& \left(P_{+} \psi_{+}\right)^{a} \equiv \chi_{z}^{a} \in \Gamma\left(\Omega_{\Sigma}^{(1,0)} \otimes \Phi^{*}\left(T_{+}^{1,0} X\right)\right) \\
& \left(\bar{P}_{+} \psi_{+}\right)^{a} \equiv \chi^{a} \in \Gamma\left(\Phi^{*}\left(T_{+}^{0,1} X\right)\right) \\
& \left(P_{-} \psi_{-}\right)^{a} \equiv \lambda_{\bar{z}}^{a} \in \Gamma\left(\Omega_{\Sigma}^{(0,1)} \otimes \Phi^{*}\left(T_{-}^{1,0} X\right)\right) \\
& \left(\bar{P}_{-} \psi_{-}\right)^{a} \equiv \lambda^{a} \in \Gamma\left(\Phi^{*}\left(T_{-}^{0,1} X\right)\right)
\end{aligned}
$$

\subsection{Generalized A model}

We will use the generalized A-model as our first explicit example. The BRST variation of the fields can be written down by setting the variation of $\bar{Q}_{+}$and $Q_{-}$in (3.14) to be zero.

$$
\begin{array}{ll}
\left\{Q_{A}, \phi^{a}\right\}= & \chi^{a}+\lambda^{a} \\
\left\{Q_{A}, \chi^{a}\right\}= & -i \Gamma_{+b c}^{a} \lambda^{b} \chi^{c} \\
\left\{Q_{A}, \lambda^{a}\right\}= & -i \Gamma_{-b c}^{a} \chi^{b} \lambda^{c} \\
\left\{Q_{A}, \chi_{z}^{a}\right\}= & -i \Gamma_{+b c}^{a}\left(\chi^{b}+\lambda^{b}\right) \chi_{z}^{c} \\
& -\left(\bar{P}_{+} \partial_{z} \phi\right)^{a}+i \bar{P}_{+d}^{a} H_{b c}^{d} \chi^{b} \chi_{z}^{c} \\
\left\{Q_{A}, \lambda_{\bar{z}}^{a}\right\}= & -i \Gamma_{-b c}^{a}\left(\chi^{b}+\lambda^{b}\right) \lambda_{\bar{z}}^{a} \\
& -\left(P_{-} \partial_{z} \phi\right)^{a}-i P_{-d}^{a} H_{b c}^{d} \lambda^{b} \lambda_{\bar{z}}^{c}
\end{array}
$$

After the twisting the Lagrangian becomes:

$$
\begin{aligned}
L & =2 t \int d^{2} z\left(\frac{1}{2}\left(g_{a b}+b_{a b}\right) \partial_{z} \phi^{a} \partial_{\bar{z}} \phi^{b}+i g_{a b}\left(\chi_{z}^{a} \partial_{\bar{z}} \chi^{b}+\lambda_{\bar{z}}^{a} \partial_{z} \lambda^{b}\right)\right. \\
& \left.+i\left(\Gamma_{a b c}-H_{a b c}\right) \chi_{z}^{a} \partial_{\bar{z}} \phi^{b} \chi^{c}+i\left(\Gamma_{a b c}+H_{a b c}\right) \lambda_{\bar{z}}^{a} \partial_{z} \phi^{b} \lambda^{c}+R_{+a b c d} \chi^{a} \chi_{z}^{b} \lambda_{\bar{z}}^{c} \lambda^{d}\right)
\end{aligned}
$$

We mimic the $V_{A}$ operator in Kähler A model (2.9) by virtue of the projectors.

$$
\mathcal{V}_{A}=g_{a b}\left(\chi_{z}^{a}\left(P_{+} \partial_{\bar{z}} \phi\right)^{b}+\lambda_{\bar{z}}^{a}\left(\bar{P}_{-} \partial_{z} \phi\right)^{b}\right)
$$

The BRST variations of $\left(P_{+} \partial_{\bar{z}} \phi\right)^{b}$ and $\left(\bar{P}_{-} \partial_{z} \phi\right)^{b}$ will involve the derivatives of the complex structures and can be re-expressed in terms of $\Gamma_{ \pm}$and the projectors (3.9) by using (3.4) and $J_{ \pm}=-i\left(P_{ \pm}-\bar{P}_{ \pm}\right)$. 


$$
\begin{aligned}
\left\{Q_{A},\left(P_{+} \partial \phi\right)^{b}\right\}=\partial \chi^{b}+\left(P_{+} \partial \lambda\right)^{b} & +\frac{1}{2} \Gamma_{+e c}^{d}\left(P_{+}-\bar{P}_{+}\right)_{d}^{b}\left(\chi^{c}+\lambda^{c}\right) \partial \phi^{e} \\
& -\frac{1}{2} \Gamma_{+c d}^{b}\left(\chi^{c}+\lambda^{c}\right)\left(P_{+} \partial \phi-\bar{P}_{+} \partial \phi\right)^{d} \\
\left\{Q_{A},\left(\bar{P}_{-} \partial \phi\right)^{b}\right\}=\partial \lambda^{b}+\left(\bar{P}_{-} \partial \chi\right)^{b} & -\frac{1}{2} \Gamma_{-e c}^{d}\left(P_{-}-\bar{P}_{-}\right)_{d}^{b}\left(\chi^{c}+\lambda^{c}\right) \partial \phi^{e} \\
& +\frac{1}{2} \Gamma_{-c d}^{b}\left(\chi^{c}+\lambda^{c}\right)\left(P_{-} \partial \phi-\bar{P}_{-} \partial \phi\right)^{d}
\end{aligned}
$$

Here the $\partial$ operator could be either $\partial_{z}$ or $\partial_{\bar{z}}$. Performing the BRST variations to $V$ by using (3.18) and (3.21) we obtain

$$
\begin{array}{r}
\left\{Q_{A}, \mathcal{V}_{A}\right\}=i g_{a b}\left(\left(\bar{P}_{+} \partial_{z} \phi\right)^{a}\left(P_{+} \partial_{\bar{z}} \phi\right)^{b}+\left(P_{-} \partial_{\bar{z}} \phi\right)^{a}\left(\bar{P}_{-} \partial_{z} \phi\right)^{b}\right)+g_{a b}\left(\chi_{z}^{a} \partial_{\bar{z}} \chi^{b}+\lambda_{\bar{z}}^{a} \partial_{z} \lambda^{b}\right) \\
+\left(\Gamma_{a b c}+H_{a b c}\right) \chi_{z}^{a} \partial_{\bar{z}} \phi^{b} \chi^{c}+\left(\Gamma_{a b c}-H_{a b c}\right) \lambda_{\bar{z}}^{a} \partial_{z} \phi^{b} \lambda^{c}
\end{array}
$$

The curvature term will be recovered if we use the equations of motion for $\chi_{z}$ and $\lambda_{\bar{z}}$. To visualize that the model only depends on one of the generalized complex structure one can use the following identities.

$$
\begin{aligned}
& g\left(P_{ \pm} \cdot, \bar{P}_{ \pm} \cdot\right)=\frac{1}{2} g(\cdot, \cdot)+\frac{i}{2} g\left(J_{ \pm} \cdot, \cdot\right)=\frac{1}{2} g(\cdot, \cdot)+\frac{i}{2} \omega_{ \pm}(\cdot, \cdot) \\
& g\left(\bar{P}_{ \pm} \cdot, P_{ \pm} \cdot\right)=\frac{1}{2} g(\cdot, \cdot)-\frac{i}{2} g\left(J_{ \pm} \cdot, \cdot\right)=\frac{1}{2} g(\cdot, \cdot)-\frac{i}{2} \omega_{ \pm}(\cdot, \cdot)
\end{aligned}
$$

The scalar term in (3.22) becomes

$$
g_{a b}\left(\left(\bar{P}_{+} \partial_{z} \phi\right)^{a}\left(P_{+} \partial_{\bar{z}} \phi\right)^{b}+\left(P_{-} \partial_{\bar{z}} \phi\right)^{a}\left(\bar{P}_{-} \partial_{z} \phi\right)^{b}\right)=2 g_{a b} \partial_{z} \phi^{a} \partial_{\bar{z}} \phi^{b}-i \tilde{\omega}_{a b} \partial_{z} \phi^{a} \partial_{\bar{z}} \phi^{b}
$$

where $\tilde{\omega}=\frac{1}{2}\left(\omega_{+}+\omega_{-}\right)$which appear in $\mathcal{J}_{2}$ in (A.2).

Comparing the twisted action (3.19) and (3.22) we obtain the following suggestive equation, modulo the equations of motion for $\chi_{z}$ and $\lambda_{\bar{z}}$.

$$
L=i t \int d^{2} z\left\{Q_{A}, \mathcal{V}_{A}\right\}+t \int \Phi^{*}(-i \tilde{\omega})+t \int \Phi^{*}(b)
$$

Apparently the action of the generalized A model depends on one of the generalized complex structures $\mathcal{J}_{2}$ and the pullback of the spacetime $b$ field. The topological feature of the action will be made clear in the next section. 


\subsection{Generalized B model}

The generalized B model has the field contents as listed in (3.17). By projecting out $\epsilon_{ \pm}$ in (3.14) the BRST variations for these fields are similarly obtained.

$$
\begin{array}{ll}
\left\{Q_{B}, \phi^{a}\right\}= & \chi^{a}+\lambda^{a} \\
\left\{Q_{B}, \chi^{a}\right\}= & -i \Gamma_{+b c}^{a} \lambda^{b} \chi^{c} \\
\left\{Q_{B}, \lambda^{a}\right\}= & -i \Gamma_{-b c}^{a} \chi^{b} \lambda^{c} \\
\left\{Q_{B}, \chi_{z}^{a}\right\}= & -i \Gamma_{+b c}^{a}\left(\chi^{b}+\lambda^{b}\right) \chi_{z}^{c} \\
& -\left(P_{+} \partial_{z} \phi\right)^{a}+i P_{+d}^{a} H_{b c}^{d} \chi^{b} \chi_{z}^{c} \\
\left\{Q_{B}, \lambda_{\bar{z}}^{a}\right\}= & -i \Gamma_{-b c}^{a}\left(\chi^{b}+\lambda^{b}\right) \lambda_{\bar{z}}^{a} \\
& -\left(P_{-} \partial_{z} \phi\right)^{a}-i P_{-d}^{a} H_{b c}^{d} \lambda^{b} \lambda_{\bar{z}}^{c}
\end{array}
$$

with $Q_{B}=\bar{Q}_{+}+\bar{Q}_{-}$. Comparing (3.18) and (3.26) we can see that the A and B model variantion laws are simply exchanged if we substitute $J_{+}$by $-J_{+}$. In generalized B model the operator in the BRST exact term is given by

$$
\mathcal{V}_{B}=g_{a b}\left(\chi_{z}^{a}\left(\bar{P}_{+} \partial_{\bar{z}} \phi\right)^{b}+\lambda_{\bar{z}}^{a}\left(\bar{P}_{-} \partial_{z} \phi\right)^{b}\right)
$$

The variations of $\left(\bar{P}_{ \pm} \partial \phi\right)^{b}$ are given by

$$
\begin{aligned}
\left\{Q_{B},\left(\bar{P}_{ \pm} \partial \phi\right)^{b}\right\}=\left(\bar{P}_{ \pm}(\partial \chi+\partial \lambda)\right)^{b} & -\frac{1}{2} \Gamma_{ \pm e c}^{d}\left(P_{ \pm}-\bar{P}_{ \pm}\right)_{d}^{b}\left(\chi^{c}+\lambda^{c}\right) \partial \phi^{e} \\
& +\frac{1}{2} \Gamma_{-c d}^{b}\left(\chi^{c}+\lambda^{c}\right)\left(P_{ \pm} \partial \phi-\bar{P}_{ \pm} \partial \phi\right)^{d}
\end{aligned}
$$

Note that $\bar{P}_{+} \chi=\chi$ and $\bar{P}_{-} \lambda=\lambda$. Again the $\partial$ could be either $\partial_{z}$ or $\partial \bar{z}$.

The Lagrangian after the twisting is given by

$$
\begin{aligned}
L & =2 t \int d^{2} z\left(\frac{1}{2}\left(g_{a b}+b_{a b}\right) \partial_{z} \phi^{a} \partial_{\bar{z}} \phi^{b}+i g_{a b}\left(\chi_{z}^{a} \partial_{\bar{z}} \chi^{b}+\lambda_{\bar{z}}^{a} \partial_{z} \lambda^{b}\right)\right. \\
& \left.+i\left(\Gamma_{a b c}-H_{a b c}\right) \chi_{z}^{a} \partial_{\bar{z}} \phi^{b} \chi^{c}+i\left(\Gamma_{a b c}+H_{a b c}\right) \lambda_{\bar{z}}^{a} \partial_{z} \phi^{b} \lambda^{c}+R_{+a b c d} \chi^{a} \chi_{z}^{b} \lambda^{c} \lambda_{\bar{z}}^{d}\right)
\end{aligned}
$$

In order to determine the pullback term we compute $\left\{Q, \mathcal{V}_{B}\right\}$.

$$
\begin{array}{r}
\left\{Q_{B}, \mathcal{V}_{B}\right\}=i g_{a b}\left(\left(P_{+} \partial_{z} \phi\right)^{a}\left(\bar{P}_{+} \partial_{\bar{z}} \phi\right)^{b}+\left(P_{-} \partial_{\bar{z}} \phi\right)^{a}\left(\bar{P}_{-} \partial_{z} \phi\right)^{b}\right)+g_{a b}\left(\chi_{z}^{a} \partial_{\bar{z}} \chi^{b}+\lambda_{\bar{z}}^{a} \partial_{z} \lambda^{b}\right) \\
+\left(\Gamma_{a b c}+H_{a b c}\right) \chi_{z}^{a} \partial_{\bar{z}} \phi^{b} \chi^{c}+\left(\Gamma_{a b c}-H_{a b c}\right) \lambda_{\bar{z}}^{a} \partial_{z} \phi^{b} \lambda^{c}(
\end{array}
$$


In deriving this we have used the equations of motion of the fermionic fields. Note that (3.22) and (3.30) are almost the same except for the scalar kinetic terms. This will result in the different GCS dependence. Namely,

$$
L=i t \int d^{2} z\left\{Q_{B}, \mathcal{V}_{B}\right\}+t \int \Phi^{*}(i \delta \omega)+t \int \Phi^{*}(b)
$$

where $\delta \omega=\frac{1}{2}\left(\omega_{+}-\omega_{-}\right)$appearing in $\mathcal{J}_{1}($ A.2). Contrary to the generalized A model, the generalized B model depends on $\mathcal{J}_{1}$. At first sight the results (3.25) (3.31) seem nice and confirm our original guess. A second thought, however, reveals the issue that neither of $b-i \tilde{\omega}$ and $b+i \delta \omega$ is closed. The consequence of this is that under small coordinate repaprametrization the variation of the pullback will be nonvanishing and proportional to $H$ [16]. One way to solve this issue is to appeal to the GCG [15]. Working in generalized B model, we assume the pure spinor S $_{1}$ associated with TGC structure $\mathcal{J}_{1}$ can be put into the following form:

$$
\begin{array}{r}
\mathrm{S}_{1}=\exp (b+\beta) \\
-\bar{\beta}=b \mp i \omega_{ \pm}-\gamma_{ \pm}
\end{array}
$$

where $d \beta=0$ and the multiplication in the exponential is the wedge product. A direct but lengthy computation shows, in generalized B model,

$$
L=i t \int d^{2} z\left\{Q_{B}, \mathcal{V}_{B}+\frac{1}{2} \gamma_{+a b} \chi_{z}^{a} \partial_{\bar{z}} \phi^{b}-\frac{1}{2} \gamma_{-a b} \lambda_{\bar{z}}^{a} \partial_{z} \phi^{b}\right\}+t \int \Phi^{*}(\bar{\beta})
$$

We refer the interested readers to [15] for more details about this construction. Alternatively one could simply say that without this construction the model is topological in the sense that the worldsheet metric is irrelevant and the puckback term only depends on the homotopy class of the embedding.

\section{Conclusion and Discussion}

In this paper we study the topological twisted models with $H$-flux. We explicitly expand the $N=(2,2)$ worldsheet action with bi-Hermitian target spaces and twist the action. We found that the generalized twisted models have many similar features to the Kähler twisted models. For example, the action can always be written as a sum of a BRST exact 
term and some pullback terms, from which the geometric dependence of the topological models can be read off. The generalized A/B model depends only on one of the twisted generalized complex structures $\mathcal{J}_{2} / \mathcal{J}_{1}$.

Although it is very powerful to construct interesting examples of topological field theories by "twisting" the spins of the fields, some topological constraints for anomaly cancellations always come with it. Recently people have tried to construct the topological models for generalized geometries by using Batalin-Vilkovisky formalism to get around this limitation[12].

Another advantage of the twisted models is that it makes explicit the studying the mirror symmetry, in this case, of the non-Kähler spaces. The lacking of the non-Kähler examples, however, is a long-standing problem along this direction. Although the "generalized Kähler" examples provided in [14] are not twisted by $H$-field, it would still be very interesting to study the topological models for those geometries. Another interesting problem is to generalize the usual Kähler quotients to obtain explicit bi-Hermitian examples. We would like to visit these problems in the future.

Acknowledgments: Thanks go to Alessandro Tomasiello for very useful conversations and Anton Kapustin for commemts. We would also like to thank all the staff, organizers, speakers, and participants of Strings 2006, where the project was first initiated, for hospitality and the free food. WYC received support from the DOE under contract DE-AC03-76SF00515.

After the first version of the paper appeared on Arxiv, we received valuable comments from Roberto Zucchini, who had been working on the same problem and went even further than we did. We are grateful to him for kindly pointing out many mistakes in our previous version.

\section{A Appendix: Generalized complex geometry}

In the appendix we give a short summary of the definitions of (twisted) generalized complex structure (GC or TGC for short). Let $M$ be an even dimensional manifold and $H$ be a closed 3 -form on $M$. The twisted Dorfman backet $\circ$ is defined as a binary operation 
on the sections of $T M \oplus T^{*} M$.

$$
(X \oplus \zeta) \circ(Y \oplus \eta)=[X, Y] \oplus\left(\mathcal{L}_{X} \eta-\imath_{Y} d \zeta+\imath_{Y} \imath_{X} H\right)
$$

where $X, Y \in \Gamma(T M)$ and $\zeta, \eta \in \Gamma\left(T^{*} M\right)$. The bundle $T M \oplus T^{*} M$ has a metric $h$ with $(n, n)$ signature defined by an inner product for the sections in $T M \oplus T^{*} M$.

A TGC-structure on $M$ is an endomorphism $\mathcal{J}$ on $T M \oplus T^{*} M$ such that

(1) $\mathcal{J}^{2}=-1$

(2) $h(\cdot, \cdot)=h(\mathcal{J} \cdot, \mathcal{J} \cdot)$

(3) The $i$-eigenbundle of $\mathcal{J}$ is closed (or involutive) with respect to the twisted Dorfman bracket. This condition is equivalent to an integrability condition for the (T)GC-structure.

Setting $H=0$ the word "twisted" is dropped everywhere and we will get the definitions for Dorfman brackets and GC-structures.

(Twisted) generalized Kähler structure consists of two commuting (T)GC-structures $\mathcal{J}_{1}$ and $\mathcal{J}_{2}$ such that $\mathcal{G}=-\mathcal{J}_{1} \mathcal{J}_{2}$ is a positive definite metric on $T M \oplus T^{*} M$.

A (twisted) generalized Kähler structure is physically relevant because it has been shown that the structure is equivalent to the bi-Hermitian geometry [7]. The two (twisted) commuting generalized complex structures $\mathcal{J}_{1}$ and $\mathcal{J}_{2}$ can be expressed in terms of the data of the bi-Hermitian geometry, namely, $\left(J_{+}, J_{-}, g, H\right)$.

$$
\mathcal{J}_{1}=\left(\begin{array}{cc}
\tilde{J} & -\alpha \\
\delta \omega & -\tilde{J}^{t}
\end{array}\right), \quad \mathcal{J}_{2}=\left(\begin{array}{cc}
\delta J & -\beta \\
\tilde{\omega} & -\delta J^{t}
\end{array}\right)
$$

where

$$
\begin{gathered}
\tilde{J}=\frac{1}{2}\left(J_{+}+J_{-}\right), \quad \beta=\frac{1}{2}\left(\omega_{+}^{-1}+\omega_{-}^{-1}\right), \quad \tilde{\omega}=\frac{1}{2}\left(\omega_{+}+\omega_{-}\right), \\
\delta J=\frac{1}{2}\left(J_{+}-J_{-}\right), \quad \alpha=\frac{1}{2}\left(\omega_{+}^{-1}-\omega_{-}^{-1}\right), \quad \delta \omega=\frac{1}{2}\left(\omega_{+}-\omega_{-}\right) . \\
\omega_{ \pm}(\cdot, \cdot)=g\left(J_{ \pm} \cdot, \cdot\right)
\end{gathered}
$$

The $H$ is preserved by $J_{ \pm}$in the sense that the following constraints are satisfied and moreover it is of $(2,1)+(1,2)$ type with respect to both $J_{ \pm}$.

$$
\begin{aligned}
& H(X, Y, Z)=H\left(J_{ \pm} X, J_{ \pm} Y, Z\right)+H\left(J_{ \pm} X, Y, J_{ \pm} Z\right)+H\left(X, J_{ \pm} Y, J_{ \pm} Z\right) \\
& H\left(J_{ \pm} X, J_{ \pm} Y, J_{ \pm} Z\right)=H\left(J_{ \pm} X, Y, Z\right)+H\left(X, J_{ \pm} Y, Z\right)+H\left(X, Y, J_{ \pm} Z\right)
\end{aligned}
$$


The following identity is useful in deriving equations.

$$
H(X, Y, Z)=\mp d \omega_{ \pm}\left(J_{ \pm} X, J_{ \pm} Y, J_{ \pm} Z\right)
$$

\section{References}

[1] E. Witten, "Topological quantum field theory," Commun. Math. Phys. 117, 353 (1988).

[2] E. Witten, "Mirror manifolds and topological field theory," arXiv:hep-th/9112056.

[3] B. Zumino, "Supersymmetry And Kahler Manifolds," Phys. Lett. B 87, 203 (1979).

[4] S. J. . Gates, C. M. Hull and M. Roček, "Twisted Multiplets And New Supersymmetric Nonlinear Sigma Models," Nucl. Phys. B 248, 157 (1984).

[5] A. Kapustin and Y. Li, "Topological sigma-models with H-flux and twisted generalized complex manifolds," arXiv:hep-th/0407249.

[6] N. Hitchin, "Generalized Calabi-Yau manifolds," Quart. J. Math. Oxford Ser. 54, 281 (2003) [arXiv:math.dg/0209099].

[7] M. Gualtieri, "Generalized complex geometry," arXiv:math.dg/0401221.

[8] M. Zabzine, "Hamiltonian perspective on generalized complex structure," Commun. Math. Phys. 263, 711 (2006) [arXiv:hep-th/0502137].

[9] A. Bredthauer, U. Lindstrom, J. Persson and M. Zabzine, "Generalized Kaehler geometry from supersymmetric sigma models," arXiv:hep-th/0603130.

[10] R. Zucchini, "A topological sigma model of biKaehler geometry," JHEP 0601, 041 (2006) [arXiv:hep-th/0511144].

[11] U. Lindstrom, M. Rocek, R. von Unge and M. Zabzine, "Generalized Kaehler manifolds and off-shell supersymmetry," arXiv:hep-th/0512164.

[12] V. Pestun, "Topological strings in generalized complex space," arXiv:hepth/0603145. 
[13] S. Chiantese, F. Gmeiner and C. Jeschek, "Mirror symmetry for topological sigma models with generalized Kaehler geometry," Int. J. Mod. Phys. A 21, 2377 (2006) [arXiv:hep-th/0408169].

[14] W.-Y. Chuang, S. Kachru and A. Tomasiello, "Complex / symplectic mirrors," arXiv:hep-th/0510042.

[15] R. Zucchini, "The biHermitian topological sigma model," arXiv:hep-th/0608145.

[16] R. Zucchini, Private comments on the previous version. 